\title{
Modeling and Control of Flexible Hydraulic Robotic Arm
}

\author{
Li Dai ${ }^{1, a}$, Yao $\mathrm{Wu}^{2}, \quad$ Jian Wang ${ }^{1}$, Yungong $\mathrm{Li}^{1}, \quad$ Yu Liu ${ }^{1}$ \\ 1. School of Mechanical Engineering \& Automation, Northeastern University, Shenyang 100819, \\ China \\ 2. Shuguang Engineering Technology Department, Liaohe Petroleum Exploration Bureau, Panjin \\ 124109, China \\ a: Idai@me.neu.edu.cn
}

Key words: Flexible hydraulic robotic arm, Multi-body dynamic, PD control, Modeling

\begin{abstract}
Flexible hydraulic robotic arm is a complicated system which coupled by mechanics and hydraulics. It is widely applied in all kinds of large engineering equipments, such as concrete pump truck, bridge monitor truck, arm frame of crane, etc. The arm system of the hydraulic robotic arm is a multi-body system with redundant freedom, strong nonlinear, coupled with rigid and flexible characters. So it is of great theoretic value and real engineering significance to study the arm system of the robotic arm. In this theme, the movement of flexible hydraulic robotic arm and hydraulic cylinders are seperately analyzed with flexible multi-body dynamics, and the mechanical hydraulic dynamic model of the driving system and the arm system is built with Lagrange Equation and Virtual Work Theory. And the dynamic differential equation is built with the driving force of the hydraulic cylinder as the main force. With the track programming and the optimization method, the dynamic converse problem of the arm end track is researched, so as to get the optimized rotation angle when the arm end reaches the expected point. By using the PD control theory, without decoupling and rank-decreasing, only with feed back from the hydraulic system to realize the close loop control of the arm end position, pose and movement, the relationship between the hydraulic system and the end position \& pose is studied, so that the flexible distortion is reduced and the libration is restrained. What's more, the simulation model of the mechanical arms is built by the dynamic simulation software. The simulation result prove that the movement equation built by this way can clearly describe each dynamic character of the mechanical arms.
\end{abstract}

\section{Introduction}

With the development of the economic construct in China, the large engineering equipments is widely applied, such as concrete pump truck, bridge monitor truck, arm frame of crane, etc. There are all composed of flexible hydraulic robotic arm, and the arm system is a multi-body system with redundant freedom, strong nonlinear, coupled with rigid and flexible characters [1]. So it is of great theoretic value and real engineering significance to study the arm system of the robotic arm.

According to the information from domestic and overseas articles, the study on the flexible hydraulic robotic arm just limits to the movement of the arm system and corresponding software application $[2,3,4]$. The influence from the dynamic characters of the arms to the arm end track are not considered, which limits the control of the arm system very much [5].

In this article, the arm system of the flexible hydraulic robotic arm is considered as the study object. The mechanical hydraulic dynamic model of the driving system and the arm system is built with Lagrange Equation and Virtual Work Theory [7]. With the track programming and the 
optimization method, the dynamic converse problem of the arm end track is researched, so as to get the optimized rotation angle when the arm end reaches the expected point. By using the PD control theory, the relationship between the hydraulic system and the end position \& pose is studied.

\section{Dynamic Analysis of the Flexible Hydraulic Robotic Arms}

The Dynamic Analysis of Each Arm. Supposing each arm is homogeneous [6], the length of arm $i$ is $l_{i}$, the mass is $m_{i}$, the mass center of arm $i$ is $\mathrm{R}$ and the angle between arm $i$ and the coordinate axis is $\theta_{i}$, the coordination $n$ of the arm system is showed as Fig.1.

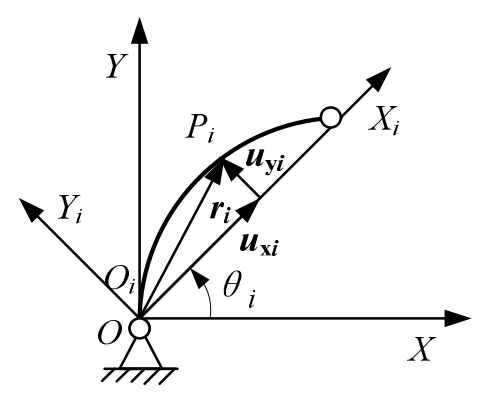

Fig. 1. The Coordination of the Arm System

According to Fig.1, we can obtain the position vector of any point $P_{i}$ on arm $i$ as follows,

$$
R_{i}=r_{i 0}+A_{i} r_{i}
$$

where $r_{i 0}=\sum_{k=1}^{i-1} A_{k} r_{k 0}$ is the position vector of $O_{i}$ in the inertial coordinate $O X Y, \boldsymbol{r}_{k 0}=\left[\begin{array}{ll}l_{k} & 0\end{array}\right]^{\mathrm{T}}$, and $\boldsymbol{A}_{\boldsymbol{i}}=\left[\begin{array}{cc}\cos \theta_{i} & -\sin \theta_{i} \\ \sin \theta_{i} & \cos \theta_{i}\end{array}\right]$ is the rotation transformation matrix from the rotational coordinate $O_{i} X_{i} Y_{i}$ to the inertial coordinate $O X Y$. And we can get $\boldsymbol{r}_{\boldsymbol{i}}=\boldsymbol{u}_{\boldsymbol{x} \boldsymbol{i}}+\boldsymbol{u}_{\boldsymbol{y} \boldsymbol{i}}$, where $\boldsymbol{u}_{\boldsymbol{x} \boldsymbol{i}}=\left[\begin{array}{ll}x_{i} & 0\end{array}\right]^{\mathrm{T}}, \quad \boldsymbol{u}_{\boldsymbol{y} \boldsymbol{i}}=\left[\begin{array}{ll}0 & v_{i}\end{array}\right]^{\mathrm{T}}$. Then $r_{i}=\left[\begin{array}{ll}x_{i} & v_{i}\end{array}\right]^{\mathrm{T}}$ is the position vector of $P_{i}$ in the rotational coordinate $O_{i} X_{i} Y_{i}$.

The velocity of point $P_{i}$ can be calculated from the first derivative of Eq.1, and can be shown as

$$
\dot{R}_{i}=\dot{r}_{i 0}+A_{i} \dot{r}_{i}+B A_{i} r_{i} \dot{\theta}_{i}
$$

in which $\quad \dot{r}_{i}=\left[\begin{array}{ll}\dot{x}_{i} & \dot{v}_{i}\end{array}\right]^{\mathrm{T}}, \quad \dot{A}_{i}=\boldsymbol{B} A_{i} \dot{\theta}_{i}$, where $\boldsymbol{B}=\left[\begin{array}{cc}0 & -1 \\ 1 & 0\end{array}\right]$.

From the rotational coordinate we can see, $\dot{x}_{1}=\frac{\partial x_{1}}{\partial t}=0$, then

$$
\dot{\boldsymbol{R}}_{\boldsymbol{i}}=\boldsymbol{A}_{\boldsymbol{i}} \dot{\boldsymbol{r}}_{\boldsymbol{i}}+\boldsymbol{B} \boldsymbol{A}_{\boldsymbol{i}} \boldsymbol{r}_{\boldsymbol{i}} \dot{\theta}_{i}+\sum_{k=1}^{i-1} \boldsymbol{B} \boldsymbol{A}_{k} \cdot \boldsymbol{r}_{k 0} \dot{\theta}_{k}
$$

Supposing the arm is homogeneous, the mass density of the arm can be shown as $\rho_{i}=\frac{m_{i}}{l_{i}}$. 
We can describe the kinetic energy of arm $i$ as follows,

$$
T_{i}=\frac{1}{2} \int_{0}^{l} \rho_{i} \dot{\boldsymbol{R}}_{\boldsymbol{i}}^{\mathrm{T}} \dot{\boldsymbol{R}}_{\boldsymbol{i}} \mathrm{d} x_{i}
$$

Then the kinetic energy of the whole system can be shown as follows,

$$
T=\frac{1}{2} \sum_{i=1}^{n} \int_{0}^{l} \rho_{i} \dot{\boldsymbol{R}}_{\boldsymbol{i}}^{\mathrm{T}} \dot{\boldsymbol{R}}_{\boldsymbol{i}} \mathrm{d} x_{i}
$$

where $n$ is the number of the arm system.

During the whole moving process of the arms, we regard the elastic distortion potential energy and the geopotential energy as the potential energy of the arms, which can be shown as,

$$
U=\frac{1}{2} \sum_{i=1}^{n} E I_{i} \int_{0}^{l_{i}}\left(\frac{\partial^{2} u_{i}}{\partial x_{i}^{2}}\right)^{2} \mathrm{~d} x_{i}+\sum_{i=1}^{n} \frac{m_{i}}{l_{i}} \mathrm{~g} \cos \theta_{i} \int_{0}^{l_{i}} u_{i} \mathrm{~d} x_{i} \quad+\sum_{i=1}^{n} m_{i} \mathrm{~g}\left(\frac{l_{i}}{2} \sin \theta_{i}+\sum_{k=1}^{i} l_{k-1} \sin \theta_{k-i}\right)
$$

where $E$ is the elastic modulus, $I$ is the inertia moment. The second item, the geopotential energy changes caused by arm distortion, is usually ignored.

The variable $v$, which is supposed to be a small distortion of the arm, is the function of time $t$ and displacement $x$. And the distortion $v_{k}$ of point $k$ on arm $k$ can be shown by linear combination of Ritz basis function $\varphi_{k p}$, described as follows,

$$
v_{k}\left(x_{k}, t\right)=\sum_{p=1}^{m_{k}} \varphi_{k p} q_{k p}
$$

where $q_{k p}$ is the corresponding general coordinate of $\varphi_{k p}, \varphi_{k p}$ is the $p^{\text {th }}$ order basis function of arm $k$, and $m_{k}$ is the order number of Ritz basis function assumed on arm $k$. Here we suppose $m_{k}=2$, thus we can get satisfying approximation. And according to the theory of cantilever, we make $\varphi_{1}, \varphi_{2}$ as the first two-steps mode function.

Solution of the Dynamic Equation. Assuming the Lagrange function $L=T-U$, and substituting corresponding items of the second Lagrange equation $\frac{d}{d t}\left(\frac{\partial L}{\partial \dot{q}_{j}}\right)-\frac{\partial L}{\partial q_{j}}=Q_{j}$ with equation Eq.6 and Eq.7, the equation can be solved. Here the general force is set as the driving force of the hydraulic cylinder and described as the driving moment, then $\boldsymbol{Q}=\left[\tau_{i}-\tau_{i-1}\right]^{\mathrm{T}}$.

According to the Fig.2, the driving moment of the cylinder can be showed as

$$
\tau_{i}=F_{i y} \cdot a_{i 2} \cos \theta_{i}+F_{i x} \cdot a_{i 2} \sin \theta_{i} .
$$

Supposing the oil pressure of the hydraulic cylinder is the same everywhere, without considering the oil leaking and the pressure lost caused by friction with the inner surface of the pipes, the driving force of the cylinder can be showed as

$$
F=m_{y} \ddot{y}+c \dot{y}+k y+f_{0} .
$$


And the displacement of cylinder i can be showed as

$$
y_{i}=r_{O B_{i}}-r_{O A_{i}}=\left[\begin{array}{c}
a_{i 2} \cos \theta_{i}+a_{i 1} \cos \theta_{i-1} \\
a_{i 2} \sin \theta_{i}+a_{i 1} \sin \theta_{i-1}
\end{array}\right] \text {. }
$$

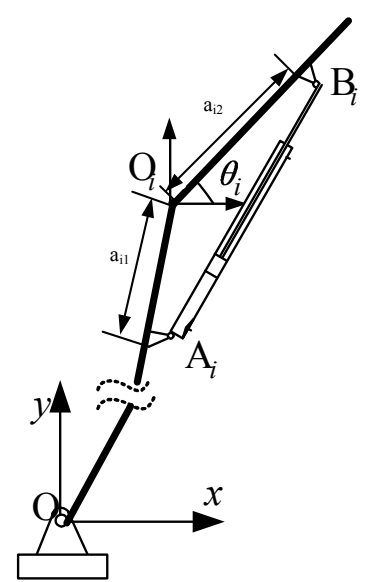

Fig. 2. The Structure Scheme of the Arm and the Cylinder

\section{PD control of the Driving System}

In order to command the end track, and to make it sure that the arms moves with the preset track, each arm must be controlled. Each part of the arm system has been analyzed in the foregoing part of this article, the dynamic differential equation can be built, based on which the position control of the arm system will realize with PD control.

Because $f_{0}$ is the initial value of spring force for the hydraulic cylinder, it is necessary to give a value while calculation. When the control rate is set as $f_{0}$, the closed-loop control of the arm and hydraulic drive system forms. Making arm $i$ as an instance, its control scheme is showed as Fig.3. By controlling the moving angle of the arms, that is controlling the spring initial value of the hydraulic driving force, the main driving force of the whole arm system can be controlled, and then the rotation angle of the arms can follow the required track.

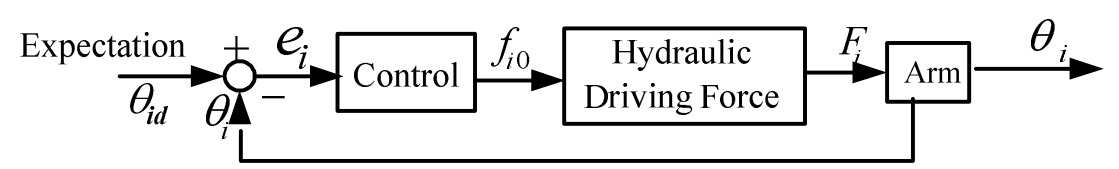

Fig. 3. The Control scheme of PD Control of the Arm System

Here the control error vector can be showed as $e_{i}=\theta_{i}-\theta_{i d}, \dot{e}_{i}=\dot{\theta}_{i}-\dot{\theta}_{i d}$, where $\theta_{i}$ is the real value of the generalized coordinate, $\theta_{i d}$ is the required value of the generalized coordinate. The PD control rate, which is applied to the initial value of the spring force for each arm, can be showed as

$f_{i 0}=k_{i p} e_{i}+k_{i d} \dot{e}_{i}$.

Then the driving force of the hydraulic cylinder for arm i can be showed as

$F_{i}=m \ddot{y}_{i}+c \dot{y}_{i}+k y_{i}+k_{i p} e_{i}+k_{i d} \dot{e}_{i}$. 
Building the Dynamic Equation. We substitute Eq.5 - Eq.12 to the second Lagrange equation,

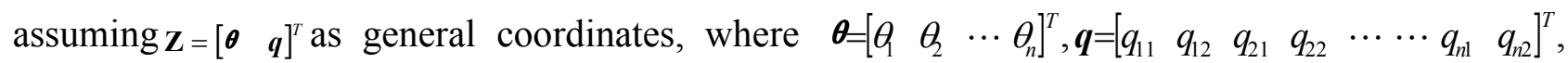
then the flexible multibody dynamics equation of arms can be shown as follows after calculating,

$$
M \ddot{Z}+V \dot{Z}^{2}+D \dot{Z}+F=0 .
$$

where $\boldsymbol{M}$ is the mass matrix, $\boldsymbol{V}$ and $\boldsymbol{D}$ are the coefficient matrixes for the $2^{\text {nd }}$ order item and the $1^{\text {st }}$ order item of the velocity.

From equation we can easily find out that the rigid movement and elastic movement exist at the same time and are strongly coupled. This is the essential characteristic of flexible multi-body dynamics.

The general coordinates can be shown as,

$$
\begin{gathered}
M_{\theta \theta} \ddot{\theta}+M_{\theta q} \ddot{q}+V_{\theta \theta} \dot{\theta}^{2}+D_{q} \dot{\theta}=F_{\theta} \\
M_{q q} \ddot{q}+K q+M_{\theta q}^{T} \ddot{\theta}+V_{q \theta} \dot{\theta}^{2}=F_{q}
\end{gathered} .
$$

\section{Analysis of an Example}

Due to the forenamed hypothesis of small distortion, the general coordinate $q$ of elastic movement is very small, then the corresponding items of the equation can be neglected. For arm system, there are rigid coupling between any two arms, also there are coupling between the rigid movement and the elastic distortion movement.

Suppose the flexible hydraulic robotic arm is 2-arms, the corresponding parameters are set as follows. For each arm, the length is $l_{1}=5 \mathrm{~m}, l_{2}=4 \mathrm{~m}$, the mass is $m_{1}=1000 \mathrm{~kg}, m_{2}=800 \mathrm{~kg}$, the initial angle is $\theta_{1}=1.48 \mathrm{rad}, \theta_{2}=1.05 \mathrm{rad}$, the initial angular velocity is $\dot{\theta}_{1}=\dot{\theta}_{2}=0$.

The control parameters are from test method, assuming $k_{p}=76, k_{d}=45$, the moving period is 5 seconds, and the step is 1000 , then the solution for the end moving track of the arm system is showed as Fig.4. From which we can see that there are some repeating fluctuation in the horizontal displacement and vertical displacement. It is the elastic distortion included in the model, built with small distortion hypothesis, which affects the angle of each arm, and then the arm end track. So it is important to consider the flexibility influence when study on the arm system.

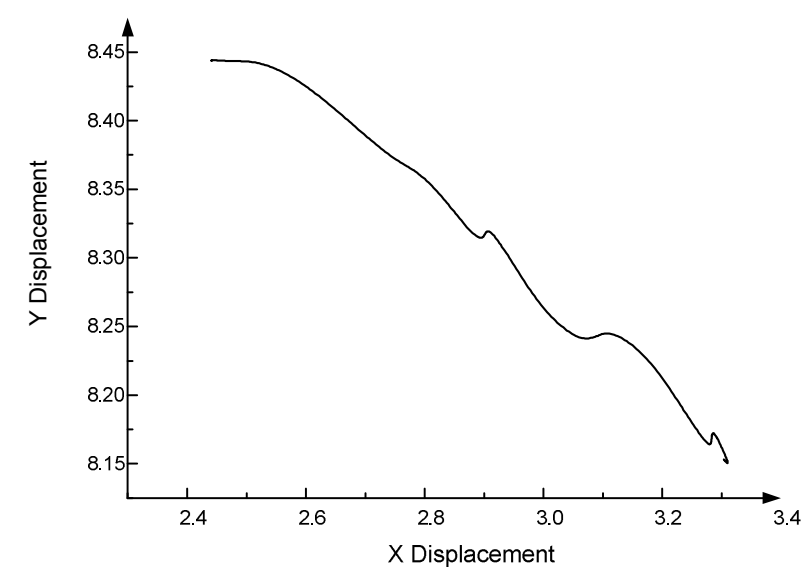

Fig. 4. The Track of the Arm End 


\section{Conclusion}

In this article, based on the recursion formula of flexible multi-body dynamics, the Lagrange Equation and the Virtual Work Theory, the dynamical differential equation of flexible hydraulic robotic arm is built. While modeling, the influence from the flexible distortion of the hydraulic cylinder to the arm end track is considered. Then the flexible multi-body dynamical equation of the arm system is deduced, numerically solved and simulated. The result shows that based on the dynamical equation of flexible arms with small distortion hypothesis, there is obvious fluctuation on the track curve of the arm end. All these changes prove the presence of the high frequency elements. That is to say, the influence from the flexible distortion to the arm end track can not be neglected.

\section{Acknowledgement}

This work is supported by the central high school is basic research traffic fee of China (Grant No. 100403004).

\section{References}

[1] Min Ye, Longxiang Xiao, Analytic mechanics. The Tianjin University Publishing Company, Tianjin, China,(2001) 140 168.

[2] Ahmed A. Shabana. Flexible multibody dynamics: review of past and recent developments. Multibody System Dynamics 1, (1997)189-222 .

[3] P. Betsch, P. Steinmann. A DAE approach to flexible multibody dynamics. Multibody System Dynamics 8, (2002) 365-389.

[4] Youfang Lu, Flexible multi-body system dynamics. The Higher Education Publishing Company, Beijing,(1996). 1-30 and 233-266.

[5] R.G. Langlois, R.J. Anderson. Multibody dynamics of very flexible damped systems. Multibody System Dynamics 3, (1999). 109-136.

[6] P. Shi, J. McPhee, G.R. Heppler. A deformation field for euler-bernoulli beams with applications to flexible multibody dynamics. Multibody System Dynamics 5, (2001).79-104.

[7] A.Y.T. Leung, Guorong Wu, Weifang Zhong. Nonlinear dynamic analysis of flexible multibody system. Acta Mechanica Solida Sinica, 17(4) (2004). 330-336. 